\title{
On existence of the prescribing $k$-curvature problem on manifolds with boundary
}

\author{
Yan He ANd Weimin Sheng
}

\begin{abstract}
In this paper, we study the problem of conformally deforming a metric to a prescribed $k$ th order symmetric function of the eigenvalues of the Schouten tensor on compact Riemannian manifolds with totally geodesic boundary. We prove the solvability of the problem and the compactness of the solution set for the case $k \geq n / 2$, provided the conformal class admits a $k$-admissible metric. These results have been proved by Gursky and Viaclovsky, Trudinger and Wang for the manifolds without boundary, and by Jin et al. and S. Chen for the locally conformally flat manifolds with boundary.
\end{abstract}

\section{Introduction}

In this paper, we study the existence of the solution to a prescribing $k$-curvature problem on manifolds with boundary and the compactness of the solution set.

Let $\left(M^{n}, g\right)$ be a smooth, compact Riemannian manifold of dimension $n \geq 3$. The Schouten tensor of $g$ is defined by

$$
A_{g}=\frac{1}{n-2}\left(\operatorname{Ric}_{g}-\frac{R_{g}}{2(n-1)} g\right),
$$

where Ric and $R$ are the Ricci and scalar curvatures of $g$, respectively. Let $[g]$ be the set of metrics conformal to $g$. For $\tilde{g}=e^{-2 u} g \in[g]$, we consider the equation

$$
\sigma_{k}^{1 / k}\left(\lambda\left(\widetilde{g}^{-1} A_{\tilde{g}}\right)\right)=f(x),
$$

where $\sigma_{k}: R^{n} \rightarrow R$ denotes the $k$ th elementary symmetric function $(1 \leq$ $k \leq n)$, and $\lambda\left(g^{-1} A_{g}\right)$ the eigenvalues of $g^{-1} A_{g} \cdot \sigma_{k}\left(\lambda\left(g^{-1} A_{g}\right)\right)$ is called $k$-curvature. The Schouten tensor transforms according to the formula

$$
A_{\tilde{g}}=\nabla^{2} u+d u \otimes d u-\frac{1}{2}|\nabla u|^{2} g+A_{g},
$$


where $\nabla u$ and $\nabla^{2} u$ denote the gradient and Hessian of $u$ with respect to $g$. Consequently, (1.1) is equivalent to

$$
\sigma_{k}^{1 / k}\left(\lambda\left(g^{-1}\left[\nabla^{2} u+d u \otimes d u-\frac{1}{2}|\nabla u|^{2} g+A_{g}\right]\right)\right)=f(x) \mathrm{e}^{-2 u} .
$$

Let $\Gamma_{k} \subset R^{n}$ denote the component of $\left\{x \in R^{n} \mid \sigma_{k}(x)>0\right\}$ containing the positive cone $\left\{x \in R^{n} \mid x_{1}>0, \ldots, x_{n}>0\right\}$ and $[g]_{k}=\left\{\tilde{g} \in[g] \mid \lambda\left(\tilde{g}^{-1} A_{\tilde{g}}\right) \in\right.$ $\left.\Gamma_{k}\right\}$. We call a metric in $[g]_{k} k$-admissible, or simply admissible. The function $u$ is called $k$-admissible, if $\mathrm{e}^{-2 u} g \in[g]_{k}$. The $k$-Yamabe problem is to find a solution of (1.2) with $f(x)=$ constant for some given metric $g$ on $M^{n}$ with $g \in \Gamma_{k}$. When $k=1$, it reduces to the classical Yamabe problem. For compact manifolds without boundary, the classical Yamabe problem (i.e., $k=1$ ) has been solved by Yamabe [1], Trudinger [2], Aubin [3] and Schoen [4]. For $k \geq 2$, the existence of the solutions to the $k$-Yamabe equation $((1.2)$ for $f(x)=$ constant $)$ has been solved for the cases $k=2, n=4$ $[5,6], k=2, n \geq 4[12], k=n / 2[7], k>n / 2[8,9]$ or for locally conformally flat manifolds [10-12,33]. The compactness of the solution set has also been proved when $k \geq n / 2$ in the above papers, and for locally conformally flat case, by [13].

For compact Riemannian manifold $\left(M^{n}, g\right)$ with non-empty smooth boundary $\partial M$, there are two classes of boundary conditions for the existence problem of equation (1.2). One is the Dirichlet boundary condition, which has been studied by Bo Guan in [14]. Another is the Neumann problem, has been studied by $\mathrm{S}$. Chen, Jin-Li-Li and $\mathrm{Li}-\mathrm{Li}[15-18]$, etc. For $k=1$, there are also several results (e.g.[19-22], etc.). Under various conditions, they derive local estimates for solutions and establish some existence results.

In this paper, we are interested in the case $k \geq n / 2$ with the Neumann boundary condition. Under the assumption that the boundary is totally geodesic, we obtain the existence of the solutions to the Neumann problem and the compactness of the solution set. In fact, we have the following

Theorem 1.1. Let $\left(M^{n}, g\right)$ be compact $n$-dimensional Riemannian manifold with totally geodesic boundary, $n \geq 3$. Assume $g$ is $k$-admissible with $k>$ $n / 2$ and $M$ is not conformally equivalent to the standard hemisphere. Then for any given smooth positive function $f \in C^{\infty}(M)$, there exists a smooth function $u \in C^{\infty}(M)$ such that the conformal metric $\tilde{g}=e^{-2 u} g$ satisfies

$$
\sigma_{k}^{\frac{1}{k}}\left(\lambda\left(\tilde{g}^{-1} A_{\tilde{g}}\right)\right)=f(x)
$$

and $M$ has totally geodesic boundary under $\tilde{g}$. In addition, the set of all such solutions is compact in the $C^{m}$-topology for any $m \geq 0$. 
Theorem 1.2. Let $\left(M^{n}, g\right)$ be a compact $n$-dimensional Riemannian manifold with totally geodesic boundary, $n \geq 4$. Assume that $g$ is $k$-admissible with $k=n / 2$ and $(M, g)$ is not conformally equivalent to $\left(S_{n}^{+}, g_{c}\right)$, where $g_{c}$ is the standard metric on the hemisphere. Then for any given smooth positive function $f \in C^{\infty}(M)$ there exists a smooth function $u \in C^{\infty}(M)$ such that the conformal metric $\tilde{g}=e^{-2 u} g$ satisfies

$$
\sigma_{k}^{\frac{1}{k}}\left(\lambda\left(\tilde{g}^{-1} A_{\tilde{g}}\right)\right)=f(x)
$$

and $M$ has totally geodesic boundary under $\tilde{g}$. In addition, the set of all such solutions is compact in the $C^{m}$-topology for any $m \geq 0$.

Here Theorem 1.1 generalizes a result in [17] where it assumes that $(M, g)$ is locally conformally flat near $\partial M$. Theorem 1.2 improves a corresponding result in [15] and [17] for the case $k=n / 2$, where $f(x) \equiv$ constant, and $(M, g)$ is locally conformally flat.

Recall that the second fundamental form $L$ of $\partial M$ with respect to $g$ is defined as

$$
L(X, Y)=-g\left(\nabla_{X} \nu, Y\right), \quad X, Y \in T_{x}(\partial M),
$$

where $T_{x}(\partial M)$ denotes the tangent space of $\partial M$ at $x, \nu$ is the unit inward normal vector field to $\partial M$ in $(M, g), \nabla$ is the Levi-Civita connection of g. A point $x \in \partial M$ is umbilic if $L(X, Y)=\tau_{g}(x) g(X, Y)$ for all $X, Y \in$ $T_{x}(\partial M)$. The boundary is called umbilic if every point of $\partial M$ is umbilic. A totally geodesic boundary is umbilic with $\tau_{g} \equiv 0$. Note that the umbilicity is conformally invariant. In fact, we have

$$
\widetilde{L}(X, Y) \mathrm{e}^{u}=\frac{\partial u}{\partial \nu} g(X, Y)+L(X, Y), \quad \text { for any } X, Y \in T_{x}(\partial M),
$$

where $\widetilde{L}$ denotes the second fundamental form of $\partial M$ with respect to $\widetilde{g}=\mathrm{e}^{-2 u} g$. When the boundary is umbilic, the above formula becomes

$$
\tau_{\widetilde{g}} \mathrm{e}^{-u}=\frac{\partial u}{\partial \nu}+\tau_{g}
$$

Especially, if $(M, g)$ has totally geodesic boundary and the conformal metric $\tilde{g}$ has totally geodesic boundary as well, then the $k$-Yamabe problem with totally geodesic boundary becomes to consider the following equation:

$$
\begin{cases}\sigma_{k}^{1 / k}\left(\lambda\left(g^{-1}\left[\nabla^{2} u+d u \otimes d u-\frac{1}{2}|\nabla u|^{2} g+A_{g}\right]\right)\right)=f(x) \mathrm{e}^{-2 u}, & \text { in } M \\ \frac{\partial u}{\partial \nu}=0, & \text { on } \partial M\end{cases}
$$


For $k>\frac{n}{2}$, we study (1.3) along the line of [8]. By use of the deformation (2.1), which is defined in [8], we may get (1.3) when $t=1$ and the equation when $t=0$ is easier to analyze. The Leray-Schauder degree, defined in $[23]([15]$ for the boundary case) is non-zero. By homotopy-invariance of the degree, the existence of the solution reduces to establish a priori estimates for $t \in[0,1]$. To prove this, we argue it by contradiction. Assuming that there exists a sequence of solutions $\left\{u_{i}\right\}$ which fails on $C^{0}$-bound, we study its blow-up. In Section 3, we prove that there are only finite blow-up points. Then we show that the super limit of solution $u_{i}$ is $+\infty$ at regular point in Section 4. Hence, in Section 5, we can obtain a better rescaled function $w_{i}$. Then by gluing two copies of $M$ along the boundary, we derive a $C_{\text {loc }}^{1,1}$ function $\widetilde{w}$ on a closed $C^{2,1}$ manifold $\widetilde{M}$. Therefore, by the argument in sections 6 and 7 of [8], we know $\left(M^{n}, \mathrm{e}^{-2 w} g\right)$ is in fact the half-plane in Euclidean space, which contradicts with the condition that the manifold is not conformally equivalent to standard hemisphere.

However, when $k=\frac{n}{2}$, the Ricci tensor is only positive semi-definite, it is not enough to prove the existence as the case $k>n / 2$. So, we need to employ another method. In [7], Trudinger and X.-J. Wang provided an another approach. By analyzing the asymptotic behavior of the solution at singular points, they prove the existence of the solutions to equation (1.2) for manifolds without boundary. By the similar argument as [7], we may glue two copies of $M$ along the boundary as above, and prove Theorem 1.2 in Section 6.

\section{Deformation and $C^{1}$ and $C^{2}$ estimates}

\subsection{Deformation}

To prove the existence of solution to the equation (1.3), we employ the following deformation which is defined in [24]:

$$
\begin{cases}\sigma_{k}^{1 / k}\left(\lambda \left(g ^ { - 1 } \left[\lambda_{k}(1-\psi(t)) g+\psi(t) A_{g}+\nabla^{2} u+d u \otimes d u\right.\right.\right. & \\ \left.\left.\left.-\frac{1}{2}|\nabla u|^{2} g\right]\right)\right)=\psi(t) f(x) \mathrm{e}^{-2 u}+(1-t)\left(\int \mathrm{e}^{-(n+1) u}\right)^{\frac{2}{n+1}}, & \text { in } M \\ \frac{\partial u}{\partial \nu}=0, & \text { on } \partial M .\end{cases}
$$

where $\psi \in C^{1}[0,1]$ satisfies $0 \leq \psi(t) \leq 1, \psi(0)=0, \psi(t)=1$ for $t \geq \frac{1}{2}$; and $\lambda_{k}=\left(\begin{array}{l}n \\ k\end{array}\right)^{-\frac{1}{k}} \operatorname{vol}(M, g)^{\frac{2}{n+1}}$. 
As [24], at $t=1(2.1)$ becomes (1.3). While at $t=0$, it becomes

$$
\begin{cases}\sigma_{k}^{1 / k}\left(\lambda\left(g^{-1}\left[\lambda_{k} g+\nabla^{2} u+d u \otimes d u-\frac{1}{2}|\nabla u|^{2} g\right]\right)\right) & \\ =\left(\int \mathrm{e}^{-(n+1) u}\right)^{\frac{2}{n+1}}, & \text { in } M \\ \frac{\partial u}{\partial \nu}=0, & \text { on } \partial M .\end{cases}
$$

It has been pointed out in [24] that the above equation has a unique solution $u(x) \equiv 0$, if $\partial M=\varnothing$. We can show it is also true in our case.

In fact, it is obvious that $u=0$ is a solution. Now we can show its uniqueness.

At the maximum point $x_{0}$ of $u$, no matter $x_{0}$ is an interior or a boundary point, it is true that $\left.\nabla u\right|_{x_{0}}=0$, and $\left.\nabla^{2} u\right|_{x_{0}}$ is negative semi-definite. In fact, if $x_{0}$ is interior point, it is easy to be understood. Now we consider the case that $x_{0}$ is boundary point. Since $\left.\frac{\partial u}{\partial \nu}\right|_{\partial M}=0$, we have $\left.\frac{\partial u}{\partial \nu}\right|_{x_{0}}=0$ and $\left.\frac{\partial^{2} u}{\partial \nu \partial x^{\alpha}}\right|_{x_{0}}=0$. It is also true that $\left.\frac{\partial u}{\partial x^{\alpha}}\right|_{x_{0}}=0$ and $\left.\frac{\partial^{2} u}{\partial x^{\alpha} \partial x^{\beta}}\right|_{x_{0}}$ is negative semidefinite, where $\left\{x^{\alpha}\right\}_{1 \leq \alpha \leq n-1}$ is a local coordinates on the boundary $\partial M$ around $x_{0}$. For $\left.\frac{\partial^{2} u}{\partial \nu^{2}}\right|_{x_{0}}$, we denote $u(x)=u\left(x^{\prime}, x^{n}\right), x_{0}=\left(x_{0}^{\prime}, 0\right)$. For fixed $x_{0}^{\prime}$, we consider $u=u\left(x_{0}^{\prime}, x^{n}\right)$ as the function of $x^{n}$. Since $u\left(x_{0}\right)=u\left(x_{0}^{\prime}, 0\right)$ is the maximum of $u\left(x_{0}^{\prime}, x^{n}\right)$ for $0 \leq x^{n}<\epsilon,\left.\frac{\partial^{2} u}{\left(\partial x^{n}\right)^{2}}\right|_{x_{0}} \leq 0$. This means that $\left.\frac{\partial^{2} u}{\partial \nu^{2}}\right|_{x_{0}}$ is nonpositive. Therefore, $\left.\nabla^{2} u\right|_{x_{0}}$ is negative semi-definite. Now at $x_{0}$ we have

$$
\begin{aligned}
\lambda_{k}\left(\begin{array}{c}
n \\
k
\end{array}\right)^{1 / k} & =\lambda_{k} \sigma_{k}^{\frac{1}{k}}\left(\lambda\left(g^{-1} \cdot g\right)\right) \\
& \geq \sigma_{k}^{1 / k}\left(\lambda\left(g^{-1}\left[\nabla^{2} u+d u \otimes d u-\frac{1}{2}|\nabla u|^{2} g+\lambda_{k} g\right]\right)\right) \\
& =\left(\int \mathrm{e}^{-(n+1) u}\right)^{\frac{2}{n+1}} .
\end{aligned}
$$

Similarly, at the minimum point of $u$, we have $\lambda_{k}\left(\begin{array}{c}n \\ k\end{array}\right)^{1 / k} \leq\left(\int \mathrm{e}^{-(n+1) u}\right)^{\frac{2}{n+1}}$. Therefore, $\lambda_{k}\left(\begin{array}{c}n \\ k\end{array}\right)^{1 / k}=\left(\int \mathrm{e}^{-(n+1) u}\right)^{\frac{2}{n+1}}$.

By Newton-MacLaurin inequality, we can immediately get $\sigma_{k}^{1 / k} \leq$ $\frac{1}{n}\left(\begin{array}{c}n \\ k\end{array}\right)^{1 / k} \sigma_{1}$. Hence,

$$
\begin{aligned}
\lambda_{k}\left(\begin{array}{c}
n \\
k
\end{array}\right)^{1 / k} & =\sigma_{k}^{1 / k}\left(\lambda\left(g^{-1}\left[\lambda_{k} g+\nabla^{2} u+d u \otimes d u-\frac{1}{2}|\nabla u|^{2} g\right]\right)\right) \\
& \leq \frac{1}{n}\left(\begin{array}{c}
n \\
k
\end{array}\right)^{1 / k} \sigma_{1}\left(\lambda\left(g^{-1}\left[\lambda_{k} g+\nabla^{2} u+d u \otimes d u-\frac{1}{2}|\nabla u|^{2} g\right]\right)\right) \\
& =\frac{1}{n}\left(\begin{array}{c}
n \\
k
\end{array}\right)^{1 / k}\left(\triangle u+\left(1-\frac{n}{2}\right)|\nabla u|^{2}+n \lambda_{k}\right) .
\end{aligned}
$$


Then

$$
\left(\frac{n}{2}-1\right) \int_{M}|\nabla u|^{2} \leq \int_{M} \triangle u=\int_{\partial M} \frac{\partial u}{\partial \nu}=0
$$

and $u \equiv$ constant $=0$.

Thus the operator

$$
\begin{aligned}
\Psi_{t}[u]= & \sigma_{k}^{1 / k}\left(\lambda\left(g^{-1}\left[\lambda_{k}(1-\psi(t)) g+\psi(t) A_{g}+\nabla^{2} u+d u \otimes d u-\frac{1}{2}|\nabla u|^{2} g\right]\right)\right) \\
& -\psi(t) f(x) \mathrm{e}^{-2 u}-(1-t)\left(\int \mathrm{e}^{-(n+1) u}\right)^{\frac{2}{n+1}}
\end{aligned}
$$

satisfies Leray-Schauder $\operatorname{degree} \operatorname{deg}\left(\Psi_{0}, \mathcal{O}_{0}, 0\right) \neq 0$ at $t=0$, where the Leray-Schauder degree is defined by $[23]\left([15]\right.$ for the boundary case) and $\mathcal{O}_{0}$ is a neighborhood of the zero solution in $\left\{u \in C^{4, \alpha}(M): u\right.$ is $k$-admissible, $\frac{\partial u}{\partial \nu}=0$ on $\left.\partial M\right\}$. When we obtain the homotopy-invariance of degree, we can derive that the Leray-Schauder degree is non-zero at $t=1$ which implies equation (1.3) is solvable.

\section{2. $C^{1}$ and $C^{2}$ estimates}

We use Fermi coordinates in a boundary neighborhood at first. In this local coordinates, we take the geodesic in the inner normal direction $\nu=\frac{\partial}{\partial x^{n}}$ parameterized by arc length, and $\left(x^{1}, \ldots, x^{n-1}\right)$ forms a local chart on the boundary. The metric can be expressed as $g=g_{\alpha \beta} d x^{\alpha} d x^{\beta}+\left(d x^{n}\right)^{2}$. The Greek letters $\alpha, \beta, \gamma, \ldots$ stand for the tangential direction indices, $1 \leq$ $\alpha, \beta, \gamma, \ldots \leq n-1$, while the Latin letters $i, j, k, \ldots$ stand for the full indices, $1 \leq i, j, k, \ldots \leq n$. For Fermi coordinates, see [25] for details. In Fermi coordinates, the half ball is defined by $\bar{E}_{r}^{+}=\left\{x_{n} \geq 0, \sum_{i} x_{i}^{2} \leq r^{2}\right\}$ and the segment on the boundary by $\Sigma_{r}=\left\{x_{n}=0, \sum_{i} x_{i}^{2} \leq r^{2}\right\}$. We denote the RHS of the first equation of $(2.1)$ by $h(x, u)$ and define

$$
\begin{aligned}
\overline{c_{\sup }}(r)= & \sup _{\bar{E}_{r}^{+}}\left(|h|+\left|\nabla_{x} h(x, u)\right|+\left|h_{z}(x, u)\right|+\left|\nabla_{x}^{2} h(x, u)\right|\right. \\
& \left.+\left|\nabla_{x} h_{z}(x, u)\right|+\left|h_{z z}(x, u)\right|+\left|\frac{\left|\nabla_{x} h(x, u)\right|}{\inf _{M} h}\right|+\left|\frac{\left|h_{z}(x, u)\right|}{\inf _{M} h}\right|\right) .
\end{aligned}
$$


Note that the constant $(1-t)\left(\int \mathrm{e}^{-(n+1) u}\right)^{\frac{2}{n+1}}$ is less than $\sigma_{k}^{1 / k}\left(\lambda\left(g^{-1}\right.\right.$ $\left.\left.\left[\lambda_{k}(1-\psi(t)) g+\psi(t) A_{g}\right]\right)\right)$. According to Theorem 1 in [28], we have

$$
\sup _{\bar{E}_{r / 2}^{+}}\left(|\nabla u|^{2}+\left|\nabla^{2} u\right|\right) \leq C_{1} \cdot \overline{c_{\sup }}(r)=C_{2} \cdot\left(1+\mathrm{e}^{-2 \inf _{\bar{E}_{r}^{+}} u}\right),
$$

where $C_{2}=C_{2}(n, g, r, f)$.

Now we can immediately get a boundary estimate on the geodesic half ball $B(x, r)=\{y \in M \mid \operatorname{dist}(x, y)<r\}$, since there is a following relationship between the half balls in Fermi coordinates and the half geodesic balls:

$$
\bar{E}_{\rho / \sqrt{2}}^{+} \subset B(x, \rho) \subset \bar{E}_{\sqrt{5} \rho}^{+} .
$$

In fact, we may assume the fermi coordinate of $y$ is $\left(y_{1}, \ldots, y_{n}\right), z$ is on the $x_{n}$-axis satisfying $\operatorname{dist}(z, y)=\operatorname{dist}\left(x_{n}\right.$-axis, $\left.y\right)$ and let $d=\operatorname{dist}(x, y), d_{0}=$ $\operatorname{dist}(x, z)$ and $d_{1}=\operatorname{dist}(z, y)$. Now for any $y \in \bar{E}_{\rho / \sqrt{2}}^{+}$, we have $d_{0}^{2}+d_{1}^{2}=\sum_{\alpha}$ $\left(y_{\alpha}\right)^{2}+\left(y_{n}\right)^{2}<\rho^{2} / 2$. Thus the triangle inequality implies $d^{2}=\operatorname{dist}(x, y)^{2}$ $\leq(\operatorname{dist}(x, z)+\operatorname{dist}(x, y))^{2} \leq 2\left(d_{0}^{2}+d_{1}^{2}\right)<\rho^{2}$. Therefore, $y \in B(x, \rho)$. On the other hand, for any $y \in B(x, \rho)$, we have $d^{2}<\rho^{2}$. Thus $d_{1}^{2}=\operatorname{dist}(z, y)^{2} \leq$ $\operatorname{dist}(x, y)^{2}=d^{2}<\rho^{2}$ and $d_{0}=\operatorname{dist}(z, x) \leq 2 \operatorname{dist}(x, y)=2 d<2 \rho$, otherwise $d \geq d_{0}-d_{1}>2 d-d=d$. Hence, $d_{0}^{2}+d_{1}^{2}<5 \rho^{2}$ and $y \in \bar{E}_{\sqrt{5} \rho}^{+}$.

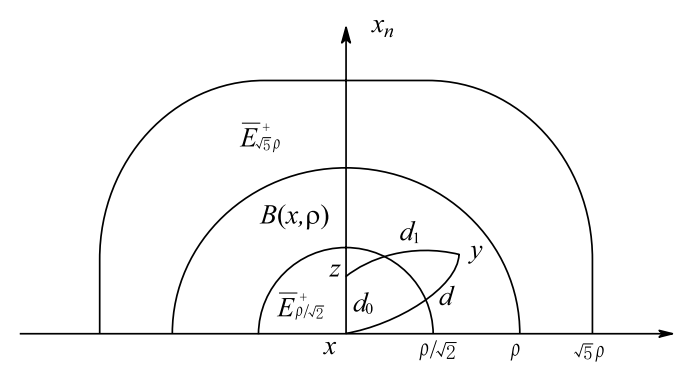

Then (2.2) implies

$$
\begin{aligned}
\sup _{B\left(x_{0}, r\right)}\left(|\nabla u|^{2}+\left|\nabla^{2} u\right|\right) & \leq \sup _{\bar{E}_{\sqrt{5} r}^{+}}\left(|\nabla u|^{2}+\left|\nabla^{2} u\right|\right) \\
& \leq C_{3}\left(1+\mathrm{e}^{-2 \inf _{\bar{E}_{2 \sqrt{5} r}^{+}} u}\right) \\
& \leq C_{3}\left(1+\mathrm{e}^{-2 \inf _{B\left(x_{0}, 2 \sqrt{10} r\right)} u}\right)
\end{aligned}
$$

where $x_{0}$ is a boundary point and $C_{3}=C_{3}(n, g, r, f)$. 
We can get interior estimate as well. Let

$$
\begin{aligned}
c_{\text {sup }}(r)= & \sup _{B\left(x_{0}, r\right)}\left(|h|+\left|\nabla_{x} h(x, u)\right|+\left|h_{z}(x, u)\right|+\left|\nabla_{x}^{2} h(x, u)\right|\right. \\
& \left.+\left|\nabla_{x} h_{z}(x, u)\right|+\left|h_{z z}(x, u)\right|+\left|\frac{\left|\nabla_{x} h(x, u)\right|}{\inf _{M} h}\right|+\left|\frac{\left|h_{z}(x, u)\right|}{\inf _{M} h}\right|\right),
\end{aligned}
$$

where $x_{0}$ is an interior point. Then by the estimates in $[26,27]$ (or Theorem 2 in [28]) we have

$$
\sup _{B\left(x_{0}, r / 2\right)}\left(|\nabla u|^{2}+\left|\nabla^{2} u\right|\right) \leq C_{4} \cdot\left(1+\mathrm{e}^{-2 \inf _{B\left(x_{0}, r\right)} u}\right),
$$

where $C_{4}=C_{4}(n, g, r, f)$.

Now we may assume that $\inf _{M} u_{i} \rightarrow-\infty$. Otherwise, by above estimate and Harnack inequality we can get the upper bound, therefore completes the proof.

There are following two possibilities.

(A) One is that the blow-up subsequence $u_{t_{i}}$ happens at $t_{i} \leq 1-\delta<1$ for $\delta>0$. We still denote it by $u_{i}$ for simplicity. Then, at the maximum point of $u_{i}$, which is either an interior point or a boundary point, we have

$$
\delta\left(\int \mathrm{e}^{-(n+1) u_{i}}\right)^{\frac{2}{n+1}} \leq \sigma_{k}^{1 / k}\left(\lambda\left(g^{-1}\left[\lambda_{k}(1-\psi(t)) g+\psi(t) A_{g}\right]\right)\right) \leq C_{0} .
$$

Then we can take $\epsilon_{i}=\mathrm{e}^{\inf _{M} u_{i}} \triangleq \mathrm{e}^{u_{i}\left(z_{i}^{0}\right)}$, where $z_{i}^{0} \in M$ is $u_{i}$ 's minimum point. Defining a map:

$$
\begin{aligned}
\mathcal{T}_{i}: \mathcal{B}\left(0, c_{0}\right) \subset T_{z_{i}^{0}} M & \rightarrow B\left(z_{i}^{0}, c_{0} \cdot \epsilon_{i}\right) \subset M \\
y & \rightarrow \exp _{z_{i}^{0}}\left(\epsilon_{i} y\right)
\end{aligned}
$$

where the metric on tangent space is $\tilde{g}_{i}=\epsilon_{i}^{-2} \mathcal{T}_{i}{ }^{*} g$ and $\mathcal{B}\left(0, c_{0}\right)$ is a geodesic ball in $\exp _{z_{i}^{0}}^{-1}(M)$ with radius $c_{0}>0$. Then we can obtain the functions with lower bounds in $\mathcal{B}\left(0, c_{0}\right)$ on the tangent space $T_{z_{i}^{0}} M: w_{i}(y)=u_{i}\left(\mathcal{T}_{i}(y)\right)-$ $\log \epsilon_{i} \geq 0$.

Furthermore, $w_{i}$ satisfies

$$
\begin{gathered}
\sigma_{k}^{1 / k}\left(\lambda\left(\tilde{g}_{i}^{-1}\left(\epsilon_{i}^{2} \lambda_{k}\left(1-\psi\left(t_{i}\right)\right) \tilde{g}_{i}+\psi\left(t_{i}\right) A_{\tilde{g}_{i}}+\nabla^{2} w_{i}+d w_{i} \otimes d w_{i}-\frac{1}{2}\left|\nabla w_{i}\right| \tilde{g}_{i} \tilde{g}_{i}\right)\right)\right) \\
=\psi\left(t_{i}\right) f\left(\mathcal{T}_{i}(y)\right) \mathrm{e}^{-2 w_{i}}+\epsilon_{i}^{2}\left(1-t_{i}\right)\left(\int \mathrm{e}^{-(n+1) u_{i}}\right)^{\frac{2}{n+1}}, \quad \text { in } \mathcal{B}\left(0, c_{0}\right) .
\end{gathered}
$$


Then by the interior and boundary estimates on $\mathcal{B}\left(0, \frac{c_{0}}{2}\right)$, we can obtain the upper bound of $w_{i}$ immediately. We then obtain the following contradiction:

$C\left(n, g, c_{0}, f\right) \leq \int_{\mathcal{B}\left(0, \frac{c_{0}}{4}\right)} \mathrm{e}^{-(n+1) w_{i}} \leq \epsilon_{i} \int_{B\left(z_{i}^{0}, c_{0} \epsilon_{i} / 2\right)} \mathrm{e}^{-(n+1) u_{i}} \leq \epsilon_{i}\left(C_{0} / \delta\right)^{\frac{n+1}{2}} \rightarrow 0$.

Therefore, we have the following boundary version of Theorem 2.1 in [24].

Lemma 2.1. For any fixed $0<\delta<1$, there is a constant $C=C(\delta, n, g, f)$ such that any $k$-admissible solution of (2.1) with $t \in[0,1-\delta]$ satisfies $\|u\|_{C^{4, \alpha}} \leq C$.

(B) So without loss of generality, we may assume that $u_{t_{i}}$ tends to $-\infty$ at the time $t_{i} \rightarrow 1$, where $u_{t_{i}}$ is the solution of $(2.1)$ at $t=t_{i}$, which will be denoted by $u_{i}$ in follows. Thus equation (2.1) is changed as

$$
\begin{cases}\sigma_{k}^{1 / k}\left(\lambda\left(g^{-1}\left(A_{g}+\nabla^{2} u+d u \otimes d u-\frac{1}{2}|\nabla u|^{2} g\right)\right)\right) & \\ =(1-t) o+f(x) \mathrm{e}^{-2 u}, & \text { in } M \\ \frac{\partial u}{\partial \nu}=0, & \text { on } \partial M .\end{cases}
$$

where $u$ is assumed to be $k$-admissible, and $o \geq 0$ is a constant.

We will obtain more exact boundary and interior estimates which are both in geodesic coordinates.

Lemma 2.2. Let $u \in C^{4}(M)$ be a k-admissible solution of (2.1) in $B(x, r)$ and $0 \leq r<1$. Then there is a constant $C=C(n, g, f)$ such that

$$
\left(\left|\nabla^{2} u\right|+|\nabla u|^{2}\right)\left(x^{\prime}\right) \leq C\left(r^{-2}+e^{-2 \inf _{B(x, 2 \sqrt{10} r)} u}\right)
$$

for all $x^{\prime} \in B(x, r)$.

Proof. If $x$ is on the boundary $\partial M$, we define a local diffeomorphism

$$
\begin{aligned}
\mathcal{O}: \mathcal{B}(0,2 \sqrt{10}) \subset T_{x} M & \rightarrow B(x, 2 \sqrt{10} r), \\
y & \rightarrow \exp _{x}(r y),
\end{aligned}
$$

where $\mathcal{B}(0,2 \sqrt{10})$ is the geodesic ball in $\exp _{x}^{-1}(M)$ with the metric $\tilde{g}=$ $r^{-2} \mathcal{O}^{*} g$. 
Let $w(y)=u(\mathcal{O}(y))-\log r$ in $\mathcal{B}(0,2 \sqrt{10})$, then $w$ satisfies

$$
\begin{gathered}
\sigma_{k}^{1 / k}\left(\lambda\left(\tilde{g}^{-1}\left[A_{\tilde{g}}+\nabla^{2} w+d w \otimes d w-\frac{1}{2}|\nabla w|_{\tilde{g}}^{2} \tilde{g}\right]\right)\right) \\
=f(\mathcal{O}(y)) \mathrm{e}^{-2 w}+r^{2}(1-t) o, \quad \text { in } \mathcal{B}(0,2 \sqrt{10}) .
\end{gathered}
$$

By a similar argument as (2.3) we can obtain

$$
\begin{aligned}
& \sup _{B(x, r)} r^{2}\left(|\nabla u|^{2}+\left|\nabla^{2} u\right|\right) \\
& \quad \leq \sup _{\mathcal{B}(0,1)}\left(|\nabla w|^{2}+\left|\nabla^{2} w\right|\right) \\
& \quad \leq C \cdot\left(e^{-2 \inf _{\mathcal{B}(0,2 \sqrt{10})} w}+1\right) \\
& \quad \leq C \cdot\left(e^{-2 \inf _{B(x, 2 \sqrt{10} r)} u} r^{2}+1\right) .
\end{aligned}
$$

Therefore,

$$
\sup _{B(x, r)}\left|\nabla^{2} u\right|(x)+|\nabla u|^{2}(x) \leq C\left(r^{-2}+e^{-2 \inf _{B(x, 2 \sqrt{10} r)} u}\right),
$$

where $C=C(n, g, f)$.

If $x$ is an interior point, the interior estimate in [8] has implied the result.

\section{Finite blow-up points}

In this section, we prove that there are finite blow-up points for prescribing $k$-curvature problem, where $k>n / 2$. By [29], we have $\operatorname{Ric}_{g} \geq \frac{(2 k-n)(n-1)}{n(k-1)}$ $\sigma_{1}\left(A_{g}\right) g$ for $k$-admissible metric $g$.

Lemma 3.1 below has been proved by Guan and Wang in [26] for the interior point. If we only focus on the boundary point, their proof also can be applied, we just notice that the ball is half ball, i.e., $B(x, \rho)$ means $B(x, \rho) \cap$ $M$. Here we omit the proof.

Lemma 3.1. There exist $\varsigma$ and $C=(\varsigma, n, g, f)$ such that any solution $u \in$ $C^{2}(B(x, \rho))$ with $\int_{B(x, \rho)} \mathrm{e}^{-n u}<\varsigma$ satisfies $\inf _{B(x, \rho / 2)} u \geq \log \rho-C$.

Similar as [24], for a given point $x \in M$, we define the mass of $x$ by

$$
m\left(\left\{u_{i}\right\} ; x\right)=\lim _{r \rightarrow 0} \limsup _{i \rightarrow \infty} \int_{B(x, r)} e^{-n u_{i}} .
$$


We also denote $\Sigma\left[\left\{u_{i}\right\}\right]=\left\{x \quad \mid \quad m\left(\left\{u_{i}\right\} ; x\right) \neq 0\right\}$.

By use of the $\epsilon$-regularity result (Lemma 3.1) and the volume comparison theorem, we may get the following propositions. See [30] for their proofs.

Proposition 3.1 (Lemma 2.3 of [30]). Given a sequence of smooth solutions of (2.4) with $\inf _{M} u_{i} \rightarrow-\infty$. Then there exists a positive constant $\mu(n, g, f, \varsigma)$ such that either $m\left(\left\{u_{i}\right\} ; x\right)=0$ or $m\left(\left\{u_{i}\right\} ; x\right) \geq \mu$.

Proposition 3.2 (Section 2 of [30]). Suppose $u_{i}$ is a blow-up sequence of (2.4), then

(1) $\Sigma\left[\left\{u_{i}\right\}\right]$ is non-empty;

(2) $\Sigma\left[\left\{u_{i}\right\}\right]$ is finite.

Corollary 3.1 (Corollary 4.7 of [8]). Let $u_{i}$ be a solution of (2.4), then

(1) when $u_{i}\left(x_{i}\right)$ converges to $-\infty$, any accumulated point of $x_{i}$ must belong to $\Sigma\left[\left\{u_{i}\right\}\right]$.

(2) for any $x_{0} \in \Sigma\left[\left\{u_{i}\right\}\right]$, there exists a subsequence $\left\{u_{k_{i}}\right\}$ and a sequence points $x_{i}$ such that $\lim _{i \rightarrow \infty} x_{i}=x_{0}$ and $\lim _{i \rightarrow \infty} u_{k_{i}}\left(x_{i}\right)=-\infty$.

Proof. (1) Let $u_{i}\left(x_{i}\right)$ be a blow-up sequence converging to $-\infty$. Since the manifold $M$ is compact, then there is a convergent subsequence $x_{i_{k}}$. Suppose $\lim _{k \rightarrow \infty} x_{i_{k}}=x_{0}$.

We assert that $x_{0}$ must be in $\Sigma\left[\left\{u_{i}\right\}\right]$. Otherwise, by Lemma 3.1, there exist constants $r_{0}$ and $J$ such that $\inf _{B\left(x_{0}, r_{0}\right)} u_{i} \geq-C$, where $i \geq J$. Furthermore, we can find $K$ such that $x_{i_{k}} \in B\left(x_{0}, r_{0}\right)$ for any $k \geq K$. Therefore, $u_{j}\left(x_{i_{k}}\right) \geq-C$ when $j \geq J$ and $k \geq K$, which contradicts the fact that $\lim _{k \rightarrow \infty} u_{i_{k}}\left(x_{i_{k}}\right)=-\infty$.

(2) Otherwise, there exists a neighborhood of $x_{0}$, for example $B\left(x_{0}, r_{0}\right)$, and a constant $C$ such that $\inf _{B\left(x_{0}, r_{0}\right)} u_{i} \geq-C$. Then

$$
m\left(\left\{u_{i}\right\} ; x_{0}\right)=\lim _{r \rightarrow 0} \limsup _{i \rightarrow \infty} \int_{B\left(x_{0}, r\right)} \mathrm{e}^{-n u_{i}} \leq \lim _{r \rightarrow 0} \int_{B\left(x_{0}, r\right)} \mathrm{e}^{n C}=0
$$

which contradicts the fact that $x_{0} \in \Sigma\left[\left\{u_{i}\right\}\right]$.

For simplicity, we still denote the subsequence by $\left\{u_{i}\right\}$. 


\section{Tend to $+\infty$ in regular set}

In this section, we prove that at any regular point $x \in M \backslash \Sigma\left[\left\{u_{i}\right\}\right], \lim \sup _{i \rightarrow \infty}$ $u_{i}(x)=+\infty$, where $\operatorname{Ric}_{g} \geq \frac{(2 k-n)(n-1)}{n(k-1)} \sigma_{1}\left(A_{g}\right) g$, when $g$ is $k$-admissible $(k>$ $n / 2)$. We prove the result by contradiction.

Suppose there is a regular point $x^{\prime}$ such that $\limsup _{i \rightarrow \infty} u_{i}\left(x^{\prime}\right)<+\infty$.

Lemma 4.1. We can find a subsequence $u_{i_{k}} \rightarrow u \in C^{\infty}\left(M \backslash \Sigma\left[\left\{u_{i}\right\}\right]\right)$, where the convergence is $C^{m}$ on compact sets away from $\Sigma\left[\left\{u_{i}\right\}\right]$.

Proof. Suppose $K$ is a compact subset in $M \backslash \Sigma\left[\left\{u_{i}\right\}\right]$. Then we can find compact sets $K^{\prime}$ and $\tilde{K}^{\prime}$, such that $K \cup\left\{x^{\prime}\right\} \subset K^{\prime} \subset \tilde{K}^{\prime}$. Since compact set $\tilde{K}^{\prime}$ is covered by finite open sets, then by Lemma 2.2, we may get $\sup _{K^{\prime}}\left|\nabla u_{i}\right| \leq$ $C_{1}$, and $\sup _{K} u_{i} \leq \sup _{K^{\prime}} u_{i} \leq \inf _{K^{\prime}} u_{i}+C_{2} \leq \limsup _{i \rightarrow \infty} u_{i}\left(x^{\prime}\right)+C_{2} \leq C_{3}$. Therefore, by regularity theory, there is a subsequence $u_{i_{k}}$ which converges uniformly to $u$ on the compact set $K$.

For simplicity we still denote the subsequence $u_{i_{k}}$ in Lemma 4.1 by $u_{i}$.

Lemma 4.2. Let $u \in C^{2}(M)$. Assume $g_{u}=\mathrm{e}^{-2 u} g$ has non-negative scalar curvature, where $\left.\frac{\partial u}{\partial \nu}\right|_{\partial M}=0$. Suppose there is a ball $B(x, \rho) \subset M$ and constants $\alpha_{0}>0$ and $B_{0}>0$ with

$$
\int_{B(x, \rho)} e^{\alpha_{0} u} d V_{g} \leq B_{0}
$$

Then there is a constant $C=C\left(n, g, \rho, \alpha_{0}, B_{0}\right)$, such that

$$
\max _{M} u \leq C
$$

Proof. This is the boundary version of Proposition 3.3 in [8]. The proof for compact manifolds without boundary is given in [8]. We just present a proof for manifolds with boundary. We denote $R_{u}$ the scalar curvature of $g_{u}$, then

$$
\frac{1}{2(n-1)} R_{u} \mathrm{e}^{-2 u}=\frac{1}{2(n-1)} R+\Delta u-\frac{n-2}{2}|\nabla u|^{2} .
$$

By the $k$-admissible $(k>n / 2)$ condition, we know that both $R_{u}$ and $R$ are positive. 
If we denote $v=\mathrm{e}^{-\frac{n-2}{2} u}$, we have

$$
\frac{n-2}{2} \frac{R_{u}}{2(n-1)} v^{\frac{4}{n-2}+1}=-\triangle v+\frac{R(n-2)}{4(n-1)} v,
$$

then

$$
\triangle v \leq C_{0} v
$$

Let $\varepsilon<\frac{2 \alpha_{0}}{n-2}$. Multiplying by $v^{-2 \varepsilon-1}$ on both sides of (4.3), and integrating by parts, we have

$$
\begin{aligned}
C_{0} \int_{M} v^{-2 \varepsilon} & \geq \int_{M} v^{-2 \varepsilon-1} \triangle v=(1+2 \varepsilon) \int_{M} v^{-2 \varepsilon-2}|\nabla v|^{2}+\int_{\partial M} v^{-2 \varepsilon-1} \frac{\partial v}{\partial \nu} \\
& =\frac{1+2 \varepsilon}{\varepsilon^{2}} \int_{M}\left|\nabla\left(v^{-\varepsilon}\right)\right|^{2} .
\end{aligned}
$$

Then, by the lower bound of the first eigenvalue $\eta_{1}$ for Neumann boundary condition (see [31]), we see that $\int_{M} v^{-2 \varepsilon}$ can be controlled by a constant depending on $\operatorname{vol}(B(x, \rho))$ and the bound of $\int_{B(x, \rho)} v^{-\varepsilon}<B_{0}^{\frac{n-2}{2 \alpha_{0}} \varepsilon}$ $\operatorname{vol}(B(x, \rho))^{1-\frac{(n-2) \varepsilon}{2 \alpha_{0}}} \triangleq A_{0}$ :

$$
\begin{aligned}
\int_{M} v^{-2 \varepsilon} \leq & \frac{\left(\int_{M} v^{-\varepsilon}\right)^{2}}{\operatorname{vol}(M)}+\frac{1}{\eta_{1}} \int_{M}\left|\nabla\left(v^{-\varepsilon}\right)\right|^{2} \leq \frac{\left(\int_{M} v^{-\varepsilon}\right)^{2}}{\operatorname{vol}(M)}+\frac{C_{0} \frac{\varepsilon^{2}}{1+2 \varepsilon}}{\eta_{1}} \int_{M} v^{-2 \varepsilon} \\
\leq & \frac{\left(\int_{B(x, \rho)} v^{-\varepsilon}+\left(\int_{B(x, \rho)^{c}} v^{-2 \varepsilon}\right)^{\frac{1}{2}} \operatorname{vol}\left(B(x, \rho)^{c}\right)^{\frac{1}{2}}\right)^{2}}{\operatorname{vol}(M)} \\
& +\frac{C_{0} \varepsilon^{2}}{\eta_{1}(1+2 \varepsilon)} \int_{M} v^{-2 \varepsilon} \\
\leq & \frac{A_{0}^{2}+A_{0} \theta \int_{B(x, \rho)^{c}} v^{-2 \varepsilon}+\frac{A_{0}}{\theta} \operatorname{vol}\left(B(x, \rho)^{c}\right)+\operatorname{vol}\left(B(x, \rho)^{c}\right) \int_{B(x, \rho)^{c}} v^{-2 \varepsilon}}{\operatorname{vol}(M)} \\
& +\frac{C_{0} \varepsilon^{2}}{\eta_{1}(1+2 \varepsilon)} \int_{M} v^{-2 \varepsilon} \\
\leq & C_{1}\left(A_{0}, \theta\right)+\left(\frac{A_{0} \theta+\operatorname{vol}\left(B(x, \rho)^{c}\right)}{\operatorname{vol}(M)}+\frac{C_{0} \varepsilon^{2}}{\eta_{1}(1+2 \varepsilon)}\right) \int_{M} v^{-2 \varepsilon} .
\end{aligned}
$$

Then by choosing a suitable constants $\varepsilon$ and $\theta$ such that

$$
\frac{A_{0} \theta+\operatorname{vol}\left(B(x, \rho)^{c}\right)}{\operatorname{vol}(M)}+\frac{C_{0} \varepsilon^{2}}{\eta_{1}(1+2 \varepsilon)}
$$


is strictly less than 1 , we can obtain the upper bound of $\int_{M} v^{-2 \varepsilon}$ which depends on $g, A_{0}, \rho$ and $\alpha_{0}$. In other words, when $\varepsilon$ is small enough, (4.1) implies a global integral upper bound of $v^{-2 \varepsilon}$.

Now we complete the proof via Green representation theorem. Note when $s<\frac{n}{n-1}$, the Green function $G$ and its gradient $|\nabla G|$ are $L^{s}$ integrable. If we denote $\frac{1}{1-\frac{1}{s}}$ by $s^{\prime}$, we wish to find a good enough $L^{s^{\prime}}$ integrable function. Denote $w=v^{-\frac{2 \varepsilon}{s^{\prime}}}$, then $w \in L^{s^{\prime}}(M)$, and

$$
\begin{aligned}
\triangle w & =-\frac{2 \varepsilon}{s^{\prime}} v^{-2 \frac{\varepsilon}{s^{\prime}}-1} \Delta v+\frac{2 \varepsilon}{s^{\prime}}\left(\frac{2 \varepsilon}{s^{\prime}}+1\right) v^{\frac{2 \varepsilon}{s^{\prime}}-2}|\nabla v|^{2} \\
& \geq-C_{2} \frac{2 \varepsilon}{s^{\prime}} w .
\end{aligned}
$$

Consequently, by Green representation theorem we have

$$
\begin{aligned}
w(a) & =-\int_{M} G(a, \cdot) \Delta w+\int_{\partial M} \frac{\partial G(a, \cdot)}{\partial \nu} w \\
& \leq C_{3} \int_{M} G(a, \cdot) w+\int_{\partial M} \frac{\partial G(a, \cdot)}{\partial \nu} w \\
& \leq C_{4}\left(\|G(a, \cdot)\| L_{L^{s}}\|w\|_{L^{s^{\prime}}}+\|\nabla G(a, \cdot)\|_{L^{s}}\|w\|_{L^{s^{\prime}}}\right) \\
& \leq C_{5}\left(n, g, A_{0}, \rho, \alpha_{0}\right) .
\end{aligned}
$$

This gives (4.2).

Proposition 4.1 (Proposition 4.6. of [8]). There is a neighborhood $B\left(x_{0}, \bar{\rho}\right)$ of $x_{0} \in \Sigma\left[\left\{u_{i}\right\}\right]$ and constant $C(n, g, f, \bar{\rho}, \varsigma)$, such that for any $x \in$ $B\left(x_{0}, \bar{\rho}\right) \backslash\left\{x_{0}\right\}$,

$$
u(x) \geq \log d_{g}\left(x, x_{0}\right)-C .
$$

Proof. Since $u_{i_{k}}$ is bounded above in some neighborhood $U$ of regular point, so

$$
\int_{U} \mathrm{e}^{\alpha u_{i_{k}}} \leq C_{1},
$$

where $\alpha$ is a constant. Then according to Lemma 4.2.

$$
\max _{M} u_{i_{k}} \leq C_{2}
$$

Let $u(x)=\lim \sup _{k \rightarrow \infty} u_{i_{k}}$ in $M$, then the limit satisfies

$$
\sup _{M \backslash \Sigma\left[\left\{u_{i}\right\}\right]} u \leq C_{2}
$$


By Volume Comparison Theorem, and Fatou Lemma,

$$
\int_{M} \mathrm{e}^{-n u} \leq \liminf _{k \rightarrow \infty} \int_{M} \mathrm{e}^{-n u_{i_{k}}}=\operatorname{vol}\left(g_{u_{i_{k}}}\right) \leq v_{0} .
$$

Hence, there exists $\bar{\rho}$ small enough such that

$$
\int_{B\left(x_{0}, 2 \bar{\rho}\right)} e^{-n u} \leq \varsigma / 2
$$

where $\varsigma$ is the constant in Lemma 3.1. Then for any point $x \in B\left(x_{0}, \bar{\rho}\right) \backslash$ $\left\{x_{0}\right\}, B\left(x, d_{g}\left(x, x_{0}\right) / 2\right) \subset B\left(x_{0}, 2 \bar{\rho}\right)$ and

$$
\int_{B\left(x, \frac{1}{2} d_{g}\left(x, x_{0}\right)\right)} \mathrm{e}^{-n u} \leq \varsigma / 2 .
$$

Since $u$ is a $C^{2}\left(B\left(x, \frac{1}{2} d\left(x, x_{0}\right)\right)\right)$ solution of $(2.4)$, by Lemma 3.1

$$
u(x) \geq \inf _{B\left(x, \frac{1}{2} d\left(x, x_{0}\right)\right)} u(x) \geq \log d_{g}\left(x, x_{0}\right)-C .
$$

Similar as the case of the manifolds without boundary in [8], for the manifolds with non-empty boundary, we can get the following propositions. Their proofs are also similar as in [8], we just notice the boundary condition $\frac{\partial u}{\partial \nu}=0$, when we take integral on the boundary. We omit their proofs.

Proposition 4.2 (Theorem 3.5. of [8]). Let $u \in C_{l o c}^{1,1}\left(A\left(\frac{1}{2} r_{1}, 2 r_{2}\right)\right)$, where $x_{0} \in M$ and $A\left(\frac{1}{2} r_{1}, 2 r_{2}\right)$ denotes the annulus $B\left(x_{0}, 2 r_{2}\right) / \overline{B\left(x_{0}, \frac{1}{2} r_{1}\right)}$, with $0<r_{1}<r_{2}$. Assume $\frac{\partial u}{\partial \nu}=0$ on the boundary $\partial M$ and $g_{u}=e^{-2 u} g$ satisfies Ric $\left(g_{u}\right)-2 \delta \sigma_{1}\left(A_{u}\right) g \geq 0$ almost everywhere in $A\left(\frac{1}{2} r_{1}, 2 r_{2}\right)$ for some $0 \leq \delta<\frac{1}{2}$. Define $\alpha_{\delta}=\frac{n-2}{1-2 \delta} \delta \leq 0$ and $p=n+2 \alpha_{\delta} \geq n$. Then for any given $\alpha \geq \alpha_{\delta}$, there is constant $C=C\left(\left(\alpha-\alpha_{\delta}\right)^{-1}, n, g\right)>0$ such that

$$
\begin{aligned}
& \int_{A\left(r_{1}, r_{2}\right)}|\nabla u|^{p} e^{\alpha u} \\
& \quad \leq C\left(\int_{A\left(\frac{1}{2} r_{1}, 2 r_{2}\right)} e^{\alpha u}|R i c|^{\frac{p}{2}}+\frac{1}{r_{1}^{p}} \int_{A\left(\frac{r_{1}}{2}, r_{2}\right)} e^{\alpha u}+\frac{1}{r_{2}^{p}} \int_{A\left(r_{1}, 2 r_{2}\right) e^{\alpha u}}\right) .
\end{aligned}
$$

Corollary 4.1 (Corollary 3.9. of [8]). Let $u \in C_{l o c}^{1,1}(M)$ satisfies $\frac{\partial u}{\partial \nu}=0$ on the boundary $\partial M$. Assume $g_{u}=e^{-2 u} g$ is $k$-admissible with $(k>n / 2)$. Suppose $\delta$ satisfies $0<\delta \leq \min \left\{\frac{1}{2}, \frac{(2 k-n)(n-1)}{2 n(k-1)}\right\}$. Define $\alpha_{\delta}=\frac{n-2}{1-2 \delta} \delta$. Then 
for any $\alpha>\alpha_{\delta}$, there exists a constant $C=C(\delta, n, g, \alpha)$ such that

$$
\left\|e^{(\alpha / p) u}\right\|_{C \gamma_{0}} \leq C\left\|e^{(\alpha / p) u}\right\|_{L^{p}}
$$

where $\gamma_{0}=\frac{2 \alpha_{\delta}}{n+2 \alpha_{\delta}}$.

Proposition 4.3 (Proposition 4.5. of [8]). Suppose $x_{0} \in \Sigma\left[\left\{u_{i}\right\}\right]$ and $u_{i}$ is a blow-up sequence near $x_{0}$. Then for any given $\theta>0$ there exists neighborhood $U$ of $x_{0}$ and a constant $C=C(\theta, n, g, f)$ such that the function $u=\lim \sup _{i \rightarrow \infty} u_{i}$ satisfies

$$
u(x) \leq(2-\theta) \log d_{g}\left(x, x_{0}\right)+C,
$$

for all $x \neq x_{0}$ in $U$.

Now from Propositions 4.1 and 4.3 we obtain a contradiction. This implies that the assumption $\limsup _{i} u_{i}\left(x^{\prime}\right)<+\infty$ for some regular point $x^{\prime}$ is impossible. Thus we have the following:

Proposition 4.4. (1) $\limsup u_{i} u_{i}=+\infty$ in $M / \Sigma\left[\left\{u_{i}\right\}\right]$.

(2) There is a subsequence $u_{i_{k}}$ converging uniformly to $+\infty$ on compact set $K \subset M / \Sigma\left[\left\{u_{i}\right\}\right]$.

Proof of (2). Note that $\lim \sup _{i} u_{i}\left(x^{\prime}\right)=+\infty$, we may suppose $\lim _{k} u_{i_{k}}\left(x^{\prime}\right)=$ $+\infty$.

There are compact sets $K^{\prime}$ and $\tilde{K}^{\prime}$, such that $K \cup\left\{x^{\prime}\right\} \subset K^{\prime} \subset \tilde{K}^{\prime}$. Applying Lemma 2.2 on $\tilde{K}^{\prime}$, we have $\sup _{K^{\prime}}\left|\nabla u_{i}\right| \leq C_{1}$ and $\inf _{K} u_{i} \geq \inf _{K^{\prime}} u_{i}$ $\geq \sup _{K^{\prime}} u_{i}-C_{2}$.

Thus for any fixed $N \in \mathbb{N}$, since $\lim _{k} u_{i_{k}}\left(x^{\prime}\right)=+\infty$ we can find $J \in \mathbb{N}$, such that $u_{i_{k}}\left(x^{\prime}\right)>N+C_{2}$ when $k>J$. Hence, $\inf _{K} u_{i_{k}} \geq u_{i_{k}}\left(x^{\prime}\right)-C_{2}>N$.

In the rest of the proof we consider the subsequence $u_{i_{k}}$ chosen above (still denoted by $u_{i}$ ) and a non-empty set $\Sigma_{0}\left[\left\{u_{i_{k}}\right\}\right] \subset \Sigma\left[\left\{u_{i}\right\}\right]$, which will be denoted by $\Sigma_{0}$ for simplicity.

\section{Complete the proof of Theorem 1.1.}

Note that $u_{i}$ satisfies $\lim _{i} u_{i}\left(x^{\prime}\right)=+\infty$, for some regular point $x^{\prime}$ in $M \backslash \Sigma_{0}$. Let

$$
w_{i}(x)=u_{i}(x)-u_{i}\left(x^{\prime}\right)
$$

We will show that $w_{i}$ converges to a $C_{\text {loc }}^{1,1}$-limit in $M \backslash \Sigma_{0}$. 


\section{Proposition 5.1. We have}

(1) $S_{w_{i}}=R_{i c} c_{w_{i}}-2 \delta \sigma_{1}\left(A_{w_{i}}\right) g$ is positive semi-definite.

(2) Let $M_{r}=M \backslash \cup_{x_{k} \in \Sigma} B\left(x_{k}, r\right)$, where $r>0$ small enough. Then we can find constants $J=J(r)$ and $C=C(n, g, f)$ such that

$$
\left|\nabla^{2} w_{i}\right|+\left|\nabla w_{i}\right|^{2} \leq C r^{-2}
$$

for all $x \in M_{r}$ and $i>J=J(r)$.

(3) The sequence $\left\{w_{i}\right\}$ has a global upper bound $\max _{M} w_{i} \leq C$ and a $L^{\infty}$ bound in $M_{r}$.

Proof. (1) $S_{w_{i}}=R i c_{w_{i}}-2 \delta \sigma_{1}\left(A_{w_{i}}\right) g=R i c_{u_{i}}-2 \delta \sigma_{1}\left(A_{u_{i}}\right) g \geq 0$.

(2) By Proposition $4.4(2)$, we can find $J(r) \in \mathbb{N}$ such that $\mathrm{e}^{-2 \inf _{M_{r / 2}} u_{i}} \leq$ $r^{-2}$ when $i>J$. Therefore, for any $x \in M_{r}$ and $i>J$, we have

$$
\begin{aligned}
& \sup _{B(x, r / 4)}\left(\left|\nabla^{2} w_{i}\right|+\left|\nabla w_{i}\right|^{2}\right) \\
& \quad=\sup _{B(x, r / 4)}\left(\left|\nabla^{2} u_{i}\right|+\left|\nabla u_{i}\right|^{2}\right) \\
& \quad \leq C_{1}\left(r^{-2}+\mathrm{e}^{-2 \inf _{M_{\sqrt{10} r / 2}} u_{i}}\right) \\
& \quad \leq 2 C_{1} r^{-2} .
\end{aligned}
$$

Since $M_{r}$ is compact, by the finite covering argument, we know that there is a constant $C=C(n, g, f)$ such that

$$
\left|\nabla^{2} w_{i}\right|+\left|\nabla w_{i}\right|^{2} \leq C r^{-2}
$$

for all $x \in M_{r}$ and $i>J=J(r)$.

(3) We may assume that $r$ is small enough and $M_{r}$ contains $x^{\prime}$. By (2) $\left|\nabla w_{i}\right|^{2}(x) \leq 2 C_{1} r^{-2}$ in $M_{r}$. Then

$$
\sup _{M_{r}} w_{i} \leq \inf _{M_{r}} w_{i}+C_{2} \leq w_{i}\left(x^{\prime}\right)+C_{2}=C_{2},
$$

where $C_{2}$ depends on $n, g, f$ and $r$. By Lemma 4.2., we obtain a global upper bound $\max _{M} w_{i} \leq C$. For the lower bound, we have

$$
\inf _{M_{r}} w_{i} \geq \sup _{M_{r}} w_{i}-C_{2} \geq w_{i}\left(x^{\prime}\right)-C_{2}=-C_{2} .
$$

Then Arzela-Ascoli theorem implies that a subsequence of $w_{i}$ (denoted by $w_{i}$ again) converges on compact sets $K \subset M \backslash \Sigma_{0}$ in $C^{1, \alpha}(K)$ where 
$\alpha \in(0,1)$. Hence, from Rademacher theorem, $\nabla^{2} w$ is well defined almost everywhere. We can obtain the following corollary immediately.

Corollary 5.1. (1) The limit $w=\lim _{i} w_{i}$ is in $C_{l o c}^{1,1}\left(M \backslash \Sigma_{0}\right)$.

(2) $S_{w}=R i c_{w}-2 \delta \sigma_{1}\left(A_{w}\right) g$ is positive semi-definite almost everywhere in $M$.

Now we may consider a doubling manifold $\widetilde{M}$ of $M$ by gluing two copies of $M$ along the boundary $\partial M$. With the given smooth Riemannian metric $g$ on $M$, there is a standard metric $\widetilde{g}$ on $\widetilde{M}$ induced from $g$. When $\partial M$ is totally geodesic in $(M, g), \widetilde{g}$ is $C^{2,1}$ on $\widetilde{M}$; see [19].

If we denote the corresponding double of $\Sigma_{0}$ by $\widetilde{\Sigma_{0}}$, we can extend $w$ to a $C_{l o c}^{1,1}\left(\widetilde{M} \backslash \widetilde{\Sigma_{0}}\right)$ function $\widetilde{w}$ as follows:

Near the boundary we take Fermi Coordinates, $\widetilde{w}$ is then defined as

$$
\widetilde{w}\left(x_{1}, \ldots, x_{n}\right)= \begin{cases}w\left(x_{1}, \ldots, x_{n}\right), & x_{n} \geq 0, \\ w\left(x_{1}, \ldots,-x_{n}\right), & x_{n} \leq 0 .\end{cases}
$$

Since $\nabla w$ is locally Lipschitz, $\nabla \widetilde{w}$ is the same. In fact, taking a geodesic convex neighborhood $\widetilde{B}(x, \widetilde{r})$ centered at any $x \in \partial M$, we may assume $p$ and $q$ are two points with $x_{n}(p) \geq 0$ and $x_{n}(q) \leq 0$. Then the geodesic connecting $p$ and $q$ is contained in $\widetilde{B}(x, \widetilde{r})$ and pass across the boundary $\partial M$. Thus there exists a point $z$ in $\widetilde{B}(x, \widetilde{r}) \cap \partial M$ such that $\widetilde{\operatorname{dist}}(p, q)=\widetilde{\operatorname{dist}}(p, z)+\widetilde{\operatorname{dist}}(z, q)$ where the distance function under metric $\widetilde{g}$ denote by $\widetilde{\operatorname{dist}}(\cdot, \cdot)$. Since $\nabla_{\frac{\partial}{\partial x^{i}}} w$ $(1 \leq i \leq n)$ is a locally Lipschitz function we know that there exist a constant $L$ such that $\left|\nabla_{\frac{\partial}{\partial x^{i}}} w(p)-\nabla_{\frac{\partial}{\partial x^{i}}} w(z)\right| \leq L \cdot \operatorname{dist}(p, z)$ and $\mid \nabla_{\frac{\partial}{\partial x^{i}}} \widetilde{w}(z)-$ $\nabla_{\frac{\partial}{\partial x^{i}}} \widetilde{w}(q) \mid \leq L \cdot \widetilde{\operatorname{dist}}(z, q)$. Therefore,

$$
\begin{aligned}
& \left|\nabla_{\frac{\partial}{\partial x^{i}}} \widetilde{w}(p)-\nabla_{\frac{\partial}{\partial x^{i}}} \widetilde{w}(q)\right| \\
& \quad \leq\left|\nabla_{\frac{\partial}{\partial x^{i}}} \widetilde{w}(p)-\nabla_{\frac{\partial}{\partial x^{i}}} \widetilde{w}(z)\right|+\left|\nabla_{\frac{\partial}{\partial x^{i}}} \widetilde{w}(z)-\nabla_{\frac{\partial}{\partial x^{i}}} \widetilde{w}(q)\right| \\
& \quad \leq L(\widetilde{\operatorname{dist}}(p, z)+\widetilde{\operatorname{dist}}(z, q)) \\
& \quad=L \cdot \widetilde{\operatorname{dist}}(p, q) .
\end{aligned}
$$

It is obviously that $\widetilde{w}$ is a $C^{1}$ function. Now we may conclude that $\widetilde{w} \in C_{\text {loc }}^{1,1}\left(\widetilde{M} \backslash \widetilde{\Sigma_{0}}\right)$. Then the following corollary is immediately.

Corollary 5.2. (1) The limit $\widetilde{w}=\lim _{i} \widetilde{w}_{i}$ is in $C_{\text {loc }}^{1,1}\left(\widetilde{M} \backslash \widetilde{\Sigma_{0}}\right)$.

(2) $S_{\widetilde{w}}=R i c_{\widetilde{w}}-2 \delta \sigma_{1}\left(A_{\widetilde{w}}\right) \widetilde{g}$ is positive semi-definite almost every where in $(\widetilde{M}, \widetilde{g})$. 
By a similar proof as it in sections 6 and 7 of [8], we can get the following proposition.

Proposition 5.2. (1) There exists an isometry

$$
\Phi:\left(\widetilde{M}_{r e g}, e^{-2 \widetilde{w}} \widetilde{g}\right) \rightarrow\left(\mathbb{R}^{n}, g_{E u c}\right),
$$

where $g_{\text {Euc }}$ is the Euclidean metric and $\widetilde{M}_{r e g}=\widetilde{M} \backslash \widetilde{\Sigma_{0}}$.

(2) $\left(M_{r e g}, e^{-2 w} g\right)$ is isometric to the half-plane in Euclidean space, where $M_{\text {reg }}=M \backslash \Sigma_{0}$.

Thus, Theorem 1.1 follows immediately, since that $(M, g)$ is assumed to be not conformally equivalent to a standard hemisphere.

\section{Proof of Theorem 1.2.}

Note that when $k=n / 2$, we cannot find a positive $\delta$ such that $\operatorname{Ric}_{\mathrm{g}} \geq$ $\delta \sigma_{1}\left(A_{g}\right) g$. Therefore, the method of the proof in Theorem 1.1 cannot be applied in the case $k=n / 2$. Nevertheless, any $k$-admissible solution $w(k \geq$ $n / 2)$ on $(M, g)$ satisfies another crucial inequality (see [7])

$$
W_{n n}+\frac{1}{n-2} \sum_{k=1}^{n} W_{k k} \geq 0
$$

where $W_{i j}=w_{i j}+w_{i} w_{j}-\frac{g_{i j}}{2}\left(\sum_{k=1}^{n} w_{k}\right)^{2}+\left(A_{g}\right)_{i j}$.

Let $u_{i}$ be a sequence of $k$-admissible solutions to equation (1.2). In [7], Trudinger and Wang consider the rescaled $k$-admissible solutions $w_{j}=u_{j}-$ $\sup _{M} u_{j}$ and prove the rescaled sequence $w_{j}$ converges in $W^{1, p}$ (for any $\left.1<p<\frac{n}{n-1}\right)$ to an admissible function $w$. Roughly speaking, if $\bar{x}$ is a blowup point of $w$, inequality (6.1) implies one side of the estimate for the limit function $w$ near $\bar{x}$ :

$$
w(x) \leq 2 \log d(x, \bar{x})+C .
$$

Furthermore, they prove

$$
w(x)=2 \log d(x, \bar{x})+o(1)
$$

where $o(1) \rightarrow 0$ when $d(x, \bar{x}) \rightarrow 0$. From (6.3), one can show that each blowup point is isolated, which implies that the number of blow-up points is finite. Combining the fact that $\mathrm{Ric}_{g} \geq 0$, by the volume comparison theorem, one 
can show as in $[8,9]$ the ratio of the volume of the geodesic ball of radius $r$ in the metric $\mathrm{e}^{-2 w} g$ with that of the Euclidean ball is non-increasing. Therefore $w$ has exactly one blow-up point 0 and the manifold $\left(M \backslash\{0\}, \mathrm{e}^{-2 w} g\right)$ is isometric to the Euclidean space, which contradicts with the assumption. Therefore there is a unform $L^{\infty}$ bound for solutions and the set of solutions is compact.

Now, similarly, when dealing with manifold with boundary we expect to prove the conformal metric $\mathrm{e}^{-2 w} g$ is in fact Euclidean metric on halfplane and get a contradiction, where $w$ is the limit function of the rescaled sequence $w_{j}=u_{j}-\sup _{M} u_{j}$ on manifold with boundary.

To this end, we double the manifold $(M, g)$. Given a smooth Riemannian metric $g$ on $M$, there is a standard metric $\widehat{g}$ on $\widehat{M}$ induced from $g$, which is glued by two copies of $M$ along the boundary $\partial M$. When $\partial M$ is totally geodesic in $(M, g)$, then $\widehat{g}$ is $C^{2,1}$ on $\widehat{M}$, see [19].

Then we extend the functions $w_{j}$ to a function $\hat{w}_{j}$ on $\widehat{M}$ as follows:

$$
\hat{w}_{j}\left(x_{1}, \cdots, x_{n}\right)= \begin{cases}w_{j}\left(x_{1}, \cdots, x_{n}\right), & x_{n} \geq 0 \\ w_{j}\left(x_{1}, \cdots,-x_{n}\right), & x_{n} \leq 0,\end{cases}
$$

where we take Fermi Coordinates near the boundary as before. We firstly verify that $\hat{w}_{j}$ satisfies the preliminary Lemmas in section 2 of [7]. However, from the boundary condition we can see that $\hat{w}_{j}$ are in fact $C^{2} k$-admissible functions on $(\widehat{M}, \widehat{g})$. We calculate under Fermi coordinates:

$$
\begin{gathered}
\lim _{x_{n} \rightarrow 0^{+}} \frac{\partial \hat{w}_{j}}{\partial x^{n}}\left(x_{1}, \ldots, x_{n}\right)=\frac{\partial w_{j}}{\partial x^{n}}\left(x_{1}, \ldots, x_{n-1}, 0\right) \\
=0=-\frac{\partial w_{j}}{\partial x^{n}}\left(x_{1}, \ldots, x_{n-1}, 0\right)=\lim _{x_{n} \rightarrow 0^{-}} \frac{\partial \hat{w}_{j}}{\partial x^{n}}\left(x_{1}, \ldots, x_{n}\right),
\end{gathered}
$$

and

$$
\lim _{x_{n} \rightarrow 0^{+}} \frac{\partial^{2} \hat{w}_{j}}{\partial\left(x^{n}\right)^{2}}\left(x_{1}, \ldots, x_{n}\right)=\lim _{x_{n} \rightarrow 0^{-}} \frac{\partial^{2} \hat{w}_{j}}{\partial\left(x^{n}\right)^{2}}\left(x_{1}, \ldots, x_{n}\right) .
$$

Thus from the $k$-admissible property of $w_{j}$ we know that $\hat{w}_{j}$ are $k$-admissible and sub-harmonic with some elliptic operator.

As a matter of fact, we may extend the definition of $k$-admissible and sub-harmonic(super-harmonic) in the viscosity sense (see [7] for details).

We call a metric $\tilde{g}=\mathcal{X} g$ is $k$-admissible if $\mathcal{X}$ is lower semi-continuous, does not equal to $\infty$, and there exists a sequence of $k$-admissible functions $\mathcal{X}_{m} \in C^{2}(M)$ such that $\mathcal{X}_{m} \rightarrow \mathcal{X}$ almost everywhere in $M$.

We say a function $v$ is super-harmonic with respect to a elliptic operator $\mathcal{L}$ if (i) $v$ is lower semi-continuous (l.s.c.); (ii) $v$ does not equal to $\infty$ in 
any open set; (iii) for any open subset $D \subset M$ and any function $h \in C^{2}(\bar{D})$ satisfying $\mathcal{L}(h)=0$ in $D$ and $h \leq v$ on $\partial D$, we have $h \leq v$ in $D$. Subharmonic functions are defined as the negative of super-harmonic ones (See p. 131 of $[32])$.

As a result, if $\hat{w}_{j}$ is $k$-admissible, then the function $\hat{v}_{j}=\mathrm{e}^{-\frac{n-2}{2} \hat{w}_{j}}$ is super-harmonic with respect to the conformal Laplace operator $\mathcal{L} \triangleq \triangle_{g}-$ $\frac{n-2}{4(n-1)} R_{g}$. The corresponding maximal (minimal) radial functions are also super-harmonic (subharmonic), where the maximal and minimal radial functions in $B_{\mathrm{R}}\left(x_{0}\right)$ are defined by

$$
\widetilde{\hat{v}}(x)=\inf \left\{\hat{v}(y): y \in \partial B_{r}\left(x_{0}\right), r=d\left(x, x_{0}\right)\right\}
$$

and

$$
\widetilde{\hat{w}}(x)=\sup \left\{\hat{w}(y): y \in \partial B_{r}\left(x_{0}\right), r=d\left(x, x_{0}\right)\right\}
$$

respectively, where $r \leq R$.

Now $\hat{w}_{j}\left(\hat{v}_{j}\right)$ and $\widehat{\widehat{\hat{w}}}_{j}\left(\widetilde{\hat{v}}_{j}\right)$ satisfying the subharmonic (super-harmonic) property, thus Lemma 2.1, Lemma 2.2, Corollary 2.1 and hence Corollary 2.2 and Corollary 2.3 in [7] still hold for $\hat{v}$ :

Lemma 6.1 (Corollary 2.2 of [7]). Let $\hat{v}$ be super-harmonic with respect to the conformal Laplacian operator $\mathcal{L}$. Then the maximal radial function $\widetilde{\hat{v}}(r)$ is locally uniformly Hölder continuous away from 0, with Hölder exponent $\alpha \in(0,1 / n)$.

Lemma 6.2 (Corollary 2.3 of [7]). Let $\hat{v}_{j}$ be a sequence of superharmonic functions which converges to $\hat{v}$ in $L^{1}\left(B_{r}(0)\right)$. Then the corresponding maximal radial functions $\widetilde{\hat{v}}_{j}(r)$ converges locally uniformly to $\widetilde{\hat{v}}(r)$.

Proof of Theorem 1.2. By use of the argument in [7] and [8], we only sketch the proof here. Suppose $x_{0, j}$ is a blow up sequence of $u_{j}$ and $\bar{x}=\lim _{j \rightarrow \infty} x_{0, j}$. Let $x_{0}^{j}$ be the maximum point of $u_{j}$. Notice that $\mathrm{e}^{-2 \sup u_{j}} f\left(x_{0}^{j}\right)=\mathrm{e}^{-2 u_{j}\left(x_{0}^{j}\right)}$ $f\left(x_{0}^{j}\right) \leq C\left(\triangle u_{j}+A_{g}\right)\left(x_{0}^{j}\right) \leq C$. Since $\sup _{M} u_{j}>-\infty, x_{0, j}$ is also a blow-up sequence of $w_{j}=u_{j}-\sup _{M} u_{j}$, and $\hat{w}_{j}\left(x_{0, j}\right) \rightarrow-\infty, \hat{v}_{j}\left(x_{0, j}\right) \rightarrow+\infty$.

Now we are going to prove the limit of $\hat{w}=\lim _{j \rightarrow \infty} \hat{w}_{j}$ satisfies (6.3). We begin with two observations: 
(1) If we denote $\hat{v}_{j}\left(x_{0, j}\right)^{\frac{1}{n-2}}$ and $\frac{1}{1-2^{-\frac{1}{n-2}}}$ by $R_{j}$ and $A_{0}$ respectively, when $j$ is large enough in $B\left(x_{0, j}, A_{0} R_{j}^{-1}\right)$ there must be some local maximum points of $\hat{v}_{j}$, denoted by $x_{j}$. Furthermore, $x_{j}$ is the local maximum points of $\hat{v}_{j}$ in $B\left(x_{j}, \frac{1}{2} \hat{v}_{j}\left(x_{j}\right)^{-\frac{1}{n-2}}\right)$ yet (see Lemma 3.2. in [7] for details).

(2) Note that the maximal and the minimal radial functions depend only on distance to the center. Thus, we may denote that

$$
\widetilde{\hat{w}}_{j}(r)=\sup \left\{\hat{w}_{j}(y): y \in \partial B_{r}\left(x_{j}\right)\right\}
$$

and

$$
\widetilde{\hat{w}}(r)=\sup \left\{\hat{w}(y): y \in \partial B_{r}(\bar{x})\right\} .
$$

In virtue of Lemmas 6.1 and 6.2 , we can obtain $\widetilde{\hat{w}}(r)=\lim _{j \rightarrow \infty} \widetilde{\hat{w}}_{j}(r)$.

Then by a similar argument in section 3 of [7], we can see that $\hat{w}$ satisfies (6.3) in $(\widehat{M}, \widehat{g})$ and singular points are isolated. Furthermore, since the Ricci curvature of $(\widehat{M}, \widehat{g})$ is still positive semi-definite, by the volume comparison theorem, there is at most one end and it is away from the singular points; the metric $\mathrm{e}^{-2 \hat{w}} \widehat{g}$, a doubling of $\mathrm{e}^{-2 w} g$, is in fact a Euclidean one (see section 7 of [8] for details). Finally, restricting the argument to manifold $M$, we can see $\left(M \backslash\{\bar{x}\}, \mathrm{e}^{-2 w} g\right)$ is just the half-plane in Euclidean space, which contradicts with the assumption. Therefore, there is a uniform $L^{\infty}$ bound for solutions and the set of solutions is compact. This completes the proof of Theorem 1.2.

\section{Acknowledgments}

The authors were supported by NSFC10771189 and 10831008. The first author (YH) would like to thank her advisor, Professor Kefeng Liu, for his support and encouragement.

\section{References}

[1] H. Yamabe, On a deformation of Riemannian structures on compact manifolds, Osaka Math. J. 12 (1960), 21-37, MR0125546, Zbl 0096.37201. 
[2] N.S. Trudinger, Remarks concerning the conformal deformation of Riemannian structures on compact manifolds, Ann. Scuola Norm. Sup. Pisa 22(3) (1968), 265-274, MR0240748, Zbl 0159.23801.

[3] T. Aubin, Equations différentielles non linéaires et problìe de Yamabe concernant la courbure scalaire, J. Math. Pures Appl. 55(9) (1976), 269-296. MR0431287, Zbl0336.53033.

[4] R. Schoen, Conformal deformation of a Riemannian metric to constant scalar curvature, J. Diff. Geom. 20 (1984), 479-495, MR0788292, Zbl 0576.53028 .

[5] A. Chang, M. Gursky and P. Yang, An equation of Monge-Amère type in conformal geometry, and four-manifolds of positive Ricci curvature, Ann. Math. 155(2) (2002), 709-787, MR1923964, Zbl 1031.53062.

[6] A. Chang, M. Gursky and P. Yang, An a priori estimate for a fully nonlinear equation on four-manifolds, J. Anal. Math. 87 (2002), 151186, MR1945280, Zbl 1067.58028.

[7] N.S. Trudinger and X-J. Wang, The intermediate case of the Yamabe problem for higher order curvatures, Int. Math. Res. Not. IMRN (13) (2010), 2437-2458.

[8] M. Gursky and J. Viaclovsky, Prescribing symmetric functions of the eigenvalues of the Ricci tensor, Ann. Math. 166 (2007), 475-531.

[9] N.S. Trudinger and X-J. Wang, On Harnack inequalities and singularities of admissible metrics in the Yamabe problem, Calc. Var. 35 (2009), 317-338.

[10] P. Guan and G. Wang, A fully nonlinear conformal flow on locally conformally flat manifolds, J. Reine Angew. Math. 557 (2003), 219238, MR1978409, Zbl 1033.53058.

[11] A. Li and Y.Y. Li, On some conformally invariant fully nonlinear equations, Comm. Pure Appl. Math. 56 (2003), 1416-1464, MR1988895, Zbl pre02002141.

[12] W.M. Sheng, N.S. Trudinger and X-J. Wang, The Yamabe problem for higher order curvatures, J. Diff. Geom. 77 (2007), 515-553.

[13] A. Li and Y.Y. Li, On some conformally invariant fully nonlinear equations. Part II: Liouville, Harnack, and Yamabe, Acta Math. 195 (2005), 117-154, MR2233687, Zbl pre05039005. 
[14] B. Guan, Conformal metrics with prescribed curvature curvature functions on manifolds with boundary, Amer. J. Math. 129(4) (2007), 915942.

[15] S. Chen, Boundary value problems for some fully nonlinear elliptic equations. Calc. Var. Partial Differential Equations 30(1) (2007), 1-15.

[16] S. Chen, Conformal Deformation on Manifolds with Boundary. Geom. Funct. Anal. 19(4) (2009), 1029-1064.

[17] Q. Jin, A. Li and Y.Y. Li, Estimates and existence results for a fully nonlinear Yamabe problem on manifolds with boundary, Calc. Var. 28 (2007), 509-543.

[18] A. Li and Y.Y. Li, A fully nonlinear version of the Yamabe problem on manifolds with boundary, J. Eur. Math. Soc. 8 (2006), 295-316.

[19] José F. Escobar, The Yamabe problem on manifolds with boundary, J. Differential Geom. 35(1) (1992), 21-84.

[20] José F. Escobar, Conformal deformation of a Riemannian metric to a scalar flat metric with constant mean curvature on the boundary, Annal. Math. 136 (1992), 1-50.

[21] Z.C. Han and Y.Y. Li, The Yamabe problem on manifolds with boundaries: existence and compactness results, Duke Math. J. 99 (1999), 489542.

[22] Z.C. Han and Y.Y. Li, The existence of conformal metrics with constant scalar curvature and constant boundary mean curvature, Commun. Anal. Geom. 8(4) (2000), 809-869.

[23] Y. Y. Li, Degree theory for second order nonlinear elliptic operators and its applications, Comm. Partial Differential Equations 14(11) (1989), 1541-1578.

[24] M. Gursky and J. Viaclovsky, Volume comparison and the $\sigma_{k}$-Yamabe problem, Adv. Math. 187(2) (2004), 447-487.

[25] A. Gray and L. Vanhecke, The volumes of tubes about curves in a Riemannian manifold, Proc. London Math. Soc. 44(3) (1982), 215-243.

[26] P. Guan and G. Wang, Local estimates for a class of fully nonlinear equations arising from conformal geometry, Int. Math. Res. Not. 26 (2003), 1413-1432, MR1976045, Zbl 1042.53021. 
[27] S. Chen, Local estimates for some fully nonlinear elliptic equations, Int. Math. Res. Not. 55 (2005), 3403-3425, MR2204639, Zbl pre05017507.

[28] Y. He and W.M. Sheng, Local estimates for some elliptic equations arising in conformal geometry, arXiv: Math.DG/1010.6115.

[29] P. Guan, J. Viaclovsky and G. Wang, Some properties of the Schouten tensor and applications to conformal geometry, Trans. Amer. Math. Soc. 355(3) (2003), 925-933.

[30] Matthew J. Gursky, Compactness of conformal metrics with integral bounds on curvature, Duke Math. J. 72(2) (1993), 339-367.

[31] P. Li and S. T. Yau, Estimates of eigenvalues of a compact Riemannian manifold, AMS, Symposium on the Geometry of the Laplace Operator, University of Hawaii at Manoa, 1979, 205-239.

[32] J. Heinonen, T. Kilpeläinen and O. Martio, Nonlinear potential theory of degenerate elliptic equations, Oxford University Press, London, 1993.

[33] J. Viaclovsky, Conformal geometry, contact geometry, and the calculus of variations, Duke Math. J. 101 (2000), 283-316, MR1738176, Zbl 0990.53035 .

Centre for Mathematical Sciences, Zhejiang University, Hangzhou 310027, CHINA

E-mail address: helenaig@hotmail.com

Department of Mathematics, Zhejiang University, Hangzhou 310027, CHINA

E-mail address: weimins@zju.edu.cn

ReCeived November 27, 2009 
